\title{
Dynamic response and fracture of composite gun tubes
}

\author{
Jerome T. Tzeng \\ US Army Research Laboratory, Weapons and \\ Materials Research Directorate, \\ Attn: AMSRL-WM-MB, Aberdeen Proving Ground, \\ MD 21005-5066, USA
}

\begin{abstract}
The fracture behavior due to dynamic response in a composite gun tube subjected to a moving pressure has been investigated. The resonance of stress waves result in very high amplitude and frequency strains in the tube at the instant and location of pressure front passage as the velocity of the projectile approaches a critical value. The cyclic stresses can accelerate crack propagation in the gun tube with an existing imperfection and significantly shorten the fatigue life of gun tubes. The fracture mechanism induced by dynamic amplification effects is particularly critical for composite overwrap barrels because of a multi-material construction, anisotropic material properties, and the potential of thermal degradation.
\end{abstract}

\section{Introduction}

When a stress wave of sufficiently large amplitude travels through a solid, it might produce fracture or accelerate the rate of propagation of an existing crack. The fracture mechanism under a transient loading condition is different from that produced by relatively static loading. This is because the velocity of the stress wave is in general much higher than the velocity of the crack propagation. Cracks might not grow in time as the stress waves pass through them. In addition, the structural deformation is non-uniform and time dependent under transient loading conditions. From a material point of view, both fracture toughness and strength can change due to various loading rates. A ductile material, such as reinforced polymer composite, might behave more brittle. The fracture toughness and strength measured in static loading conditions might not be suitable for a transient case. In this paper, stress waves due to a dynamic response in a composite wrap steel liner are modeled to study fracture behaviors of composite gun tubes. A strain energy integration approach [1] is proposed to evaluate crack propagation at the interface of the composite and steel liner. A discussion on the energy integration for prediction of the crack propagation is also included from an implementation point of view.

A very high amplitude and frequency strain, commonly referred to as dynamic strain amplification, develops in a gun tube at the passage of projectile. The phenomenon is caused by the resonance of flexural waves when the moving pressure approaches the velocity of wave propagation in the gun tube. The resonance response of an isotropic cylinder subjected to moving pressure loads has been investigated by Taylor [2], Jones and Bhuta [3], Tang [4], and Reismann [5]. More recently, Simkins [6] and Hopkins [7] investigated the dynamic response of flexural waves in steel gun tubes. Recently, Tzeng [8] extended the research on the dynamic strain effect in cylinders made of fiber-reinforced composite materials overwrap with a metal liner. The results are very applicable to lightweight composite cylinders used for gun tubes and high pressure piping systems.

\section{Analysis}

In this section, the dynamic response and fracture of a composite overwrap cylinder subjected to a moving pressure is investigated based on closed-form analytic as well as finite element methods. As a first approximation though, Love's thin shell theory can be used to derive a closed-form expression for the critical velocity. The critical velocity at which resonance occurs is greatly influenced by tube geometry and material properties. The finite element solution is obtained using a version of Dyna2d [9], which has been modified to allow accurate modeling of the moving pressure front. This approach allows modeling both the moving pressure front and the composite cylinder geometry with initial cracks in sufficient detail to simulate the actual loading conditions. Finally, an integration of strain energy density along a specific path within a cylinder is computed as an indication to determine crack propagation. 


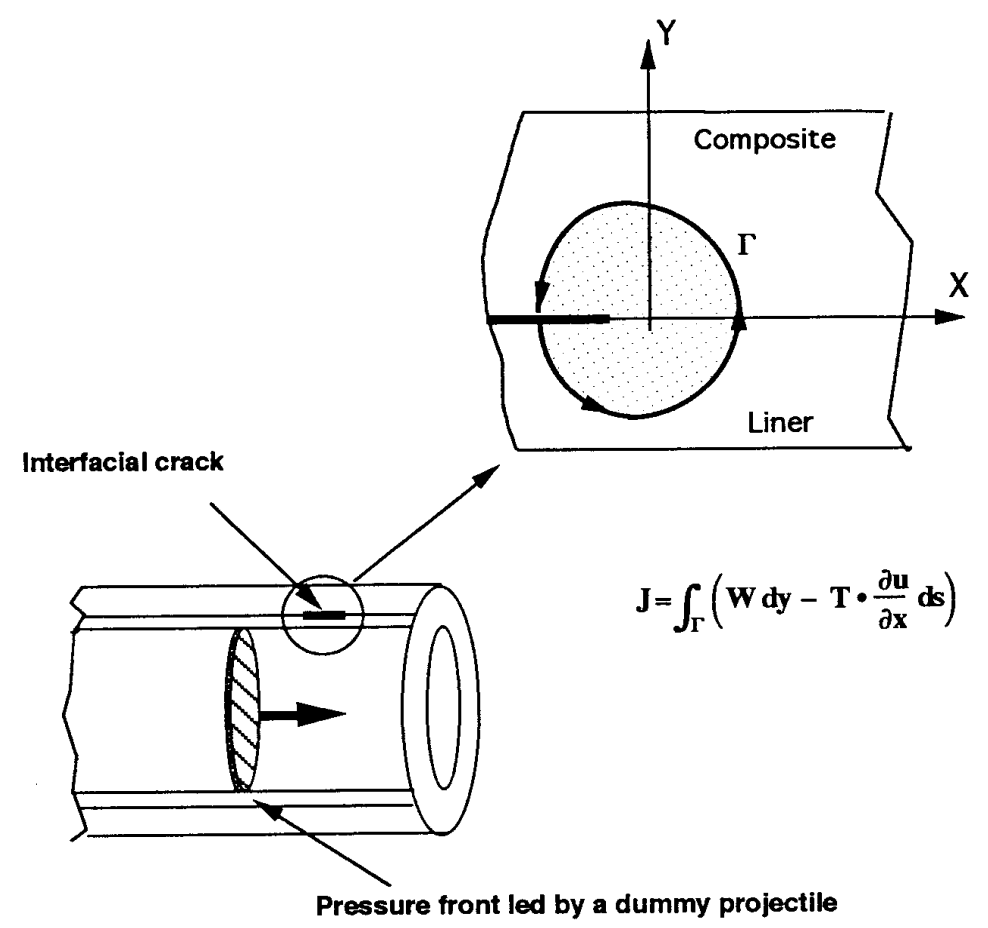

Fig. 1. Energy integration defined along an interface crack.

\subsection{Integration of energy density}

Consider a crack at the interface of the composite overwrap and steel liner as shown in Fig. 1. The strain fields near the crack tip are difficult to determine, especially in a bi-material system with anisotropy of composite laminates. Let us assume that the crack at the interface is axisymmetric and sufficiently large. Accordingly, the analysis can utilize a two-dimensional model for the far-field and crack analyses. Rice [1] proposed an approximate method known as J-integral, which bypasses the complexity of solving a boundary value problem at the crack tip. Integration along the crack tip with a path $\Gamma$ can be defined as

$$
\begin{aligned}
J= & \int_{\Gamma}\left(\omega d y-T \cdot \frac{\partial u}{\partial x} d s\right) \\
& +\int_{v}\left(\rho u \frac{\partial u}{\partial x}\right) d V,
\end{aligned}
$$

where " $\omega$ " is strain energy density and can be defined as

$$
\omega=\omega(\varepsilon)=\int_{0}^{\varepsilon} \sigma_{i j} d \varepsilon_{i j},
$$

' $T$ ' is the traction vector along the path, ' $u$ ' represents the displacement vectors, ' $d s$ ' is an element of arc length along ' $\Gamma$ ', and ' $V$ ' is a volume integration.
Atluri [10] and Doyle [11] investigated the contribution of acceleration from the internal movement of the material for a beam structure. Based on linear fracture mechanics, the J-integral is equivalent to strain energy release rate at the crack and the following expression is obtained:

$$
J=-\frac{\partial V}{\partial a}=g=g_{I}+g_{I I}+g_{I I I},
$$

$V$ is potential energy of the elastic body, and ' $a$ ' is a crack length in the body. ' $g$ ' is the total strain energy release rate resulting from three fracture modes. Accordingly, the strain energy release rate is related to stress intensity as follows:

$$
g=\frac{1-v^{2}}{E}\left(K_{I}^{2}+K_{I I}^{2}+\frac{K_{I I I}^{2}}{1-v}\right),
$$

where $K_{I}, K_{I I}$, and $K_{I I I}$, are stress intensity factors associated to different modes.

The integration of strain energy can be carried out with an implementation of the finite element technique. An overwrapped composite cylinder with an initial crack at the interface of the composite/liner is modeled in detail. Stress and strain fields at the surrounding region can be obtained through a transient analysis. Strain energy density and its integration are then calculated element by element through a specific path. 


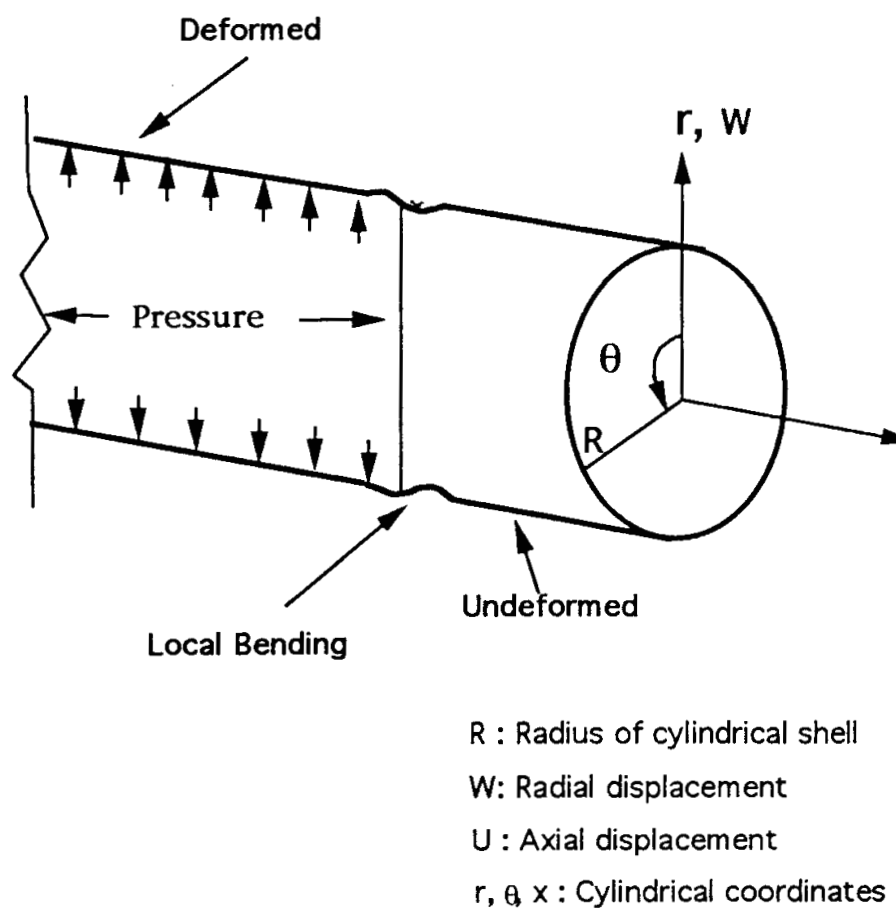

Fig. 2. Deformation of cylinder subjected to a moving pressure front.

The results will then be ready for comparison with empirical data and serve as a useful design and fabrication parameter.

\section{Dynamic response of cylinders}

The critical velocity of a flexural wave in a cylindrical tube can be obtained from Love's thin shell theory. The closed form solution is valuable in illustrating and understanding the important parameters that determine the dynamic response of the cylinder. The results can also be compared with the critical velocity values obtained using finite element techniques. The finite element values will approach the exact solution as the mesh discretization increases. Consider a thin orthotropic cylinder of radius $\mathrm{R}$ subjected to a transient pressure load (e.g., a moving internal pressure, $P$ ). Figure 2 shows the geometry, coordinate system, and pressure loading condition being considered. The governing equation for this model with a moving internal pressure front, expressed as Heaviside step function, can be shown to be given by

$$
\begin{aligned}
& m \frac{\partial^{2} W}{\partial t^{2}}+D_{x} \frac{\partial^{4} W}{\partial x^{4}}+\frac{12\left(1-v_{\theta x} v_{x \theta}\right)}{h^{2} R^{2}} D_{\theta} W \\
= & P(1-H(x-V t)),
\end{aligned}
$$

in which $W$ is the radial displacement, dependent upon time, $t$, and axial position coordinate, $x, m$ is the mass per unit length, which is equal to $\rho h, \rho$ is the density of shell material, $h$ is the thickness of the shell, $P$ is the internal pressure, and $V$ is the pressure front velocity, which is assumed constant. The shell bending stiffness in the $x-r$ and $\theta-r$ planes are given by the expressions in Eq. (6).

$$
\begin{aligned}
& D_{x}=\frac{E_{x} h^{3}}{12\left(1-v_{\theta x} v_{x \theta}\right)} \text { and } \\
& D_{\theta}=\frac{E_{\theta} h^{3}}{12\left(1-v_{\theta x} v_{x \theta}\right)},
\end{aligned}
$$

in which $E_{x}$ and $E_{\theta}$ are the effective (smeared) elastic moduli, and $v_{x \theta}$ and $v_{\theta x}$ are the effective Poisson's ratios of the composite material in the axial and circumferential directions, respectively. For a composite tube with cross-ply laminate construction, the shell bending stiffness is different in the axial and circumferential directions and is determined by the axial-to-hoop layer ratio. The loading function, $P(1-H(x-V t))$ in Eq. (5), represents the internal pressure front traveling in the axial direction with constant velocity $V$. $H(x-V t)$ is the Heaviside step function. Accordingly,

$$
\begin{aligned}
P(1-H(x-V t)) & =0 \text { when } x>V t \\
& =P \text { when } x \leqslant V t,
\end{aligned}
$$


Table 1

Effective properties of the composite wrap
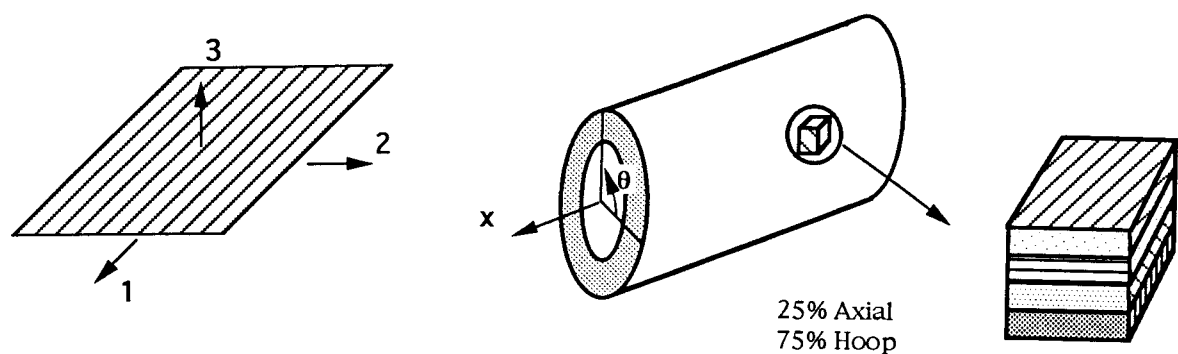

$\begin{array}{ll}E_{11}=25.0 E+06 & E_{12}=7.0 E+05 \\ E_{22}=1.2 E+06 & E_{13}=7.0 E+05 \\ E_{33}=1.2 E+06 & E_{23}=5.3 E+05 \\ v_{12}=0.33 & v_{23}=0.31 \\ v_{13}=0.33 & \end{array}$

$E_{r}=1.298 E+06 \quad G_{r x}=4.095 E+05$

$E_{x}=7.214 E+06 \quad G_{\theta x}=4.610 E+05$

$E_{\theta}=19.10 E+06 \quad G_{\theta x}=7.000 E+05$

$v_{\theta r}=0.3825$

$v_{\theta x}=0.0556$

$v_{x r}=0.338$

(unit in psi)

The critical velocity for an orthotropic cylindrical shell can be derived from the characteristic function obtained from Eq. (1) by author [8] as

$$
\begin{aligned}
V_{\mathrm{cr}, \mathrm{comp}}^{2}= & \sqrt{\frac{1}{3\left(1-v_{\theta x} v_{x \theta}\right)}}\left(\frac{h}{R}\right) \\
& \left(\frac{\sqrt{E_{\theta} E_{x}}}{\rho}\right)
\end{aligned}
$$

Equation (8) shows that the critical velocity of an orthotropic cylinder subjected to a moving pressure front is a function of the tube geometry, density, Poisson's ratios, and elastic moduli. The critical velocity increases when either of the elastic moduli increase as well as when the shell thickness to radius ratio increases. From a design point of view, a tube constructed with high stiffness and lightweight materials is preferred for dynamic loading conditions. However, Eq. (8) indicates that a larger wall thickness is required in general for the tube geometry when a high-velocity pressure front is present. It also shows that both axial and hoop moduli (e.g., $E_{\theta}$ and $E_{x}$ ) have influence on the critical velocity. Accordingly, the optimal design can be achieved by varying the laminate architecture of composite cylinders. The derived equation can also be applied to an isotropic case such as a steel liner, which has equal modulus and Poisson's ratio in all directions.

\section{Results}

The described closed-form solution can be applied accurately to a cylindrical shell under the assumption of infinite length. For a finite length cylinder with varying cross-sectional area along its length and multimaterial construction, the finite element method allows a more expedient and straightforward procedure for determining the critical velocity. Since the composite tube is a laminated construction, ideally a ply-by-ply model will yield the best result and accuracy. However, this would dictate the use of many thousands of elements in the finite element model. The level of detail, coupled with the very short time step interval required for a dynamic analysis, would lead to an unreasonably long computational time. Accordingly, a smeared properties for the composite laminate is used. These properties, which are representative of the unique layup construction of the tube, were calculated using a model developed by Alexander and Tzeng [12]. The smeared property approach allows a single finite element to contain several layers and reduces the size of the finite element model.

The cylinder used in this analysis is 100 in long with a constant wall thickness. The steel liner and composite overwrap are 0.075 in and 0.125 in thick, respectively. The effective properties for a composite tube composed of $25 \%$ axial (x-direction) and $75 \%$ hoop ( $\theta$-direction) plies are listed in Table 1 . The properties are calculated based on the use of an IM7 graphite / 8551-7 epoxy composite. The cylinder is equally divided into 400 elements along the axial direction and 10 elements through the wall thickness. There are 4 elements representing the steel liner and 6 elements representing the composite overwrap. In all, the model contains 4,000 elements. The cylinder is subjected to a moving internal pressure of 6,000 psi.

\section{Effects of projectile velocity}

Two pressure front velocities, a sub-critical velocity of 2,500 ft/s (Case 1) and a supercritical velocity of $3,500 \mathrm{ft} / \mathrm{s}$ (Case 2), were shown to demonstrate the dy- 


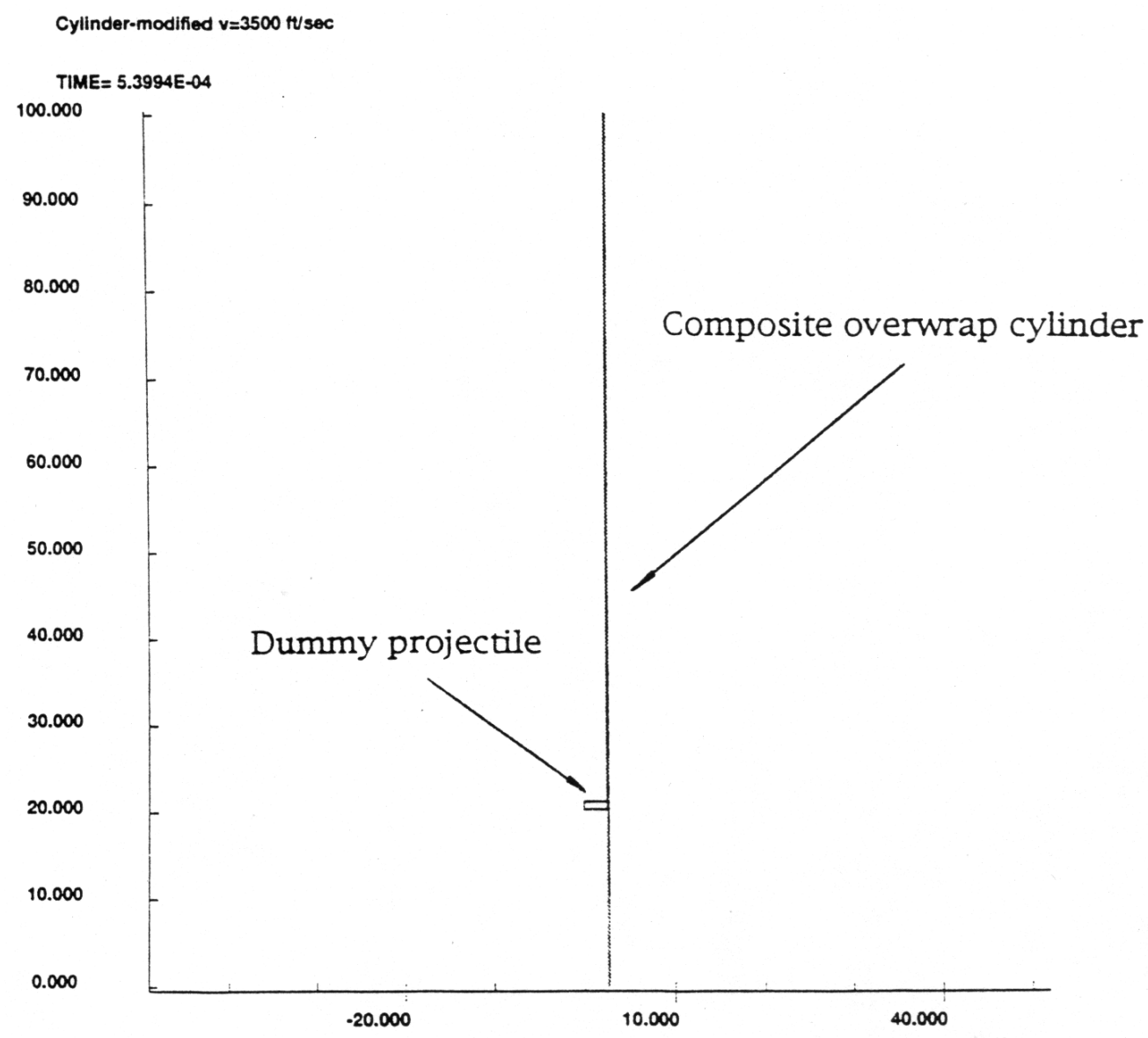

Fig. 3. FEM model of a projectile moving along a gun tube.

namic effects. The total time for the pressure front to traverse down the tube was about 3.33 and $2.43 \mathrm{~ms}$ for Cases 1 and 2, respectively. The time increment used in the analysis was of the order of s; therefore, approximately 3,500-4,000 time steps were used per analysis. The finite element models including the composite cylinder and the dummy material block are shown in Fig. 3 at a time instant when the block is about 20 in from the initial position. The block is then given an initial velocity, which is held constant throughout the analysis. Finally, because an axisymmetric model was employed, only one-half of the cylinder is shown. A fringe plot of the radial displacement in the neighborhood of the projectile with velocity of $3500 \mathrm{ft} / \mathrm{s}$ at this specific instant is shown in Fig. 4. The fringe pattern clearly shows the stress oscillation due to induced bending boundary layer stresses in the wall of the cylinder as it is subjected to a moving pressure front. There are no strain and stress waves occurring at a low velocity. The maximum displacement is located very close to the pressure front where pressure discontinuity is located. The displacement then decreases with increasing axial distance from the location of the pressure front discontinuity. The deformation is transient and cyclic with time and position.

Deformations and stresses can also be presented at a particular axial location as a time history. Figure 5 illustrates the radial deformation at the inner radius of the composite as the projectile runs through the tube. A dramatic difference is obtained in the magnitude of peak displacement as well as in temporal behavior of the oscillations as the projectile velocity changes, Case 1 versus Case 2. Before the arrival of the pressure front, the cylinder at these observed locations is basically undeformed. The small oscillations that occur just before projectile arrival are real and represent stress oscillations due the moving pressure front. Similar behavior is predicted by thin wall shell theory. It is seen that at the instant the pressure front passes, the radial displacement undergoes a rapid increase. However, for 


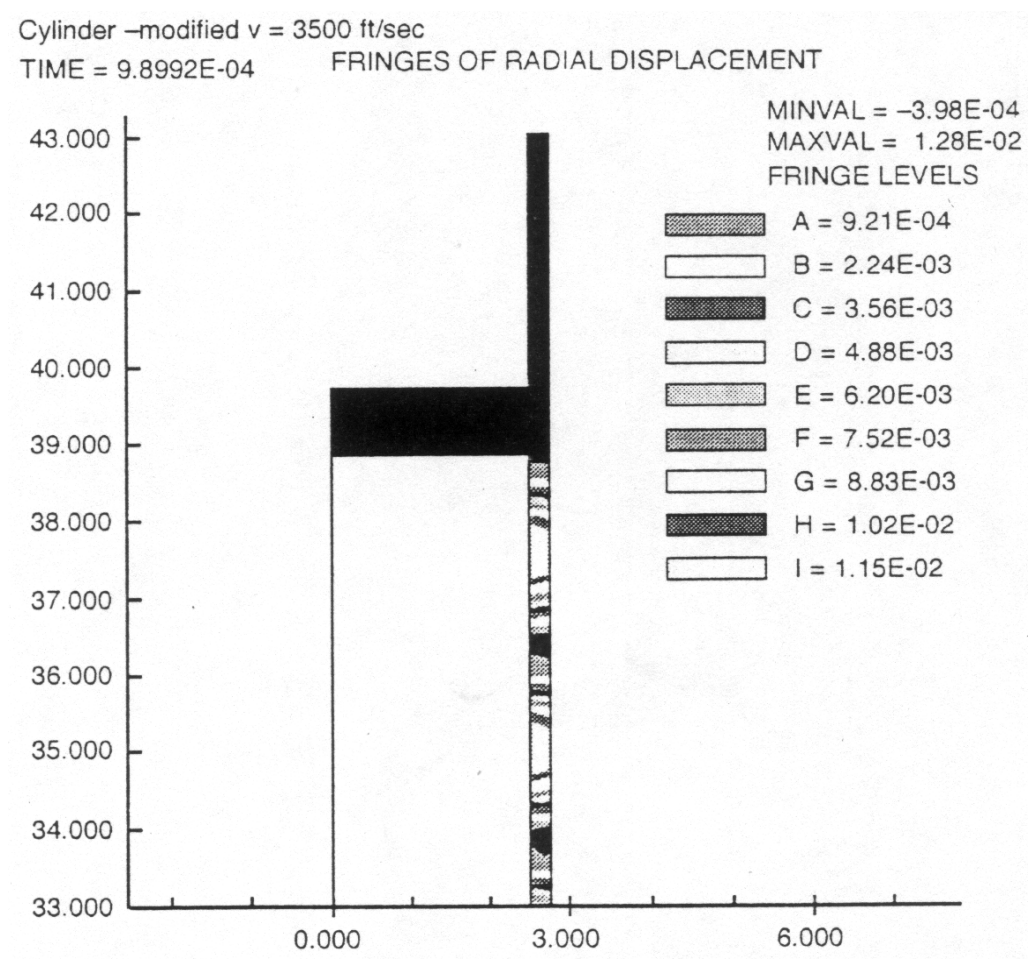

Fig. 4. Radial displacement in the vicinity of projectile passage.

sufficiently low velocities, similar to Case 1 , the displacements and stresses are still close to what would be predicted based upon a static internal pressure loading. In Case 2, a very large radial displacement occurs for this case where the pressure front velocity is 3,500 ft/s. In fact, this velocity exceeds the critical velocity of the overwrapped composite cylinder. The peak magnitude of the radial displacement is seen to be about 1.5 times the peak magnitude of the radial displacement of Case 1 where the velocity is only $2,500 \mathrm{ft} / \mathrm{s}$. As the projectile moves farther away from this axial location, the radial displacement approaches the same magnitude ( $~ 7.5 \times 10^{-3}$ in) as would be predicted by a static analysis. This is shown to be true for both Case 1 and Case 2. Since the velocity of Case 2 is above the critical velocity, the peak radial displacement is much greater than the average value with oscillation. If the projectile is accelerated from 2,500 ft/s to $3,500 \mathrm{ft} / \mathrm{s}$ gradually, the highest amplitude would happen when the critical velocity is reached. This would have resulted in a resonant condition as described previously. A similar characteristic was also found in other stress and strain components such as hoop, axial, and shear (not illustrated).

\section{Cylinders with an initial crack}

A composite overwrap tube with delamination (crack) at the interface was modeled by adding a slideline into the finite element model developed previously. It was also assumed that there was no friction at the sliding interface. Due to singularity, the stress and strain fields are not accurate in the very near region of the crack tip. Nonetheless, the far-field stress and strain are reasonably accurate and can be used to calculate strain energy density surrounding the crack. Accordingly, a superfine finite element mesh is not necessary if the dynamic response can be fully modeled. This approach can significantly reduce computation time.

Figure 6 illustrates the finite element model near the crack tip and the path of integration. Figure 7 shows the hoop strain in steel element 484 which is near the embed crack. The magnitude of strain is much larger (about double) compared to the one calculated from the model without a crack. The integration path is chosen to be along the coordinate axes to reduce computational efforts. It is also traction free along the path, which furthermore reduces the complexity of the calculation. The integration can be performed element by element along the path. The strain energy density is calculated 
Case 1

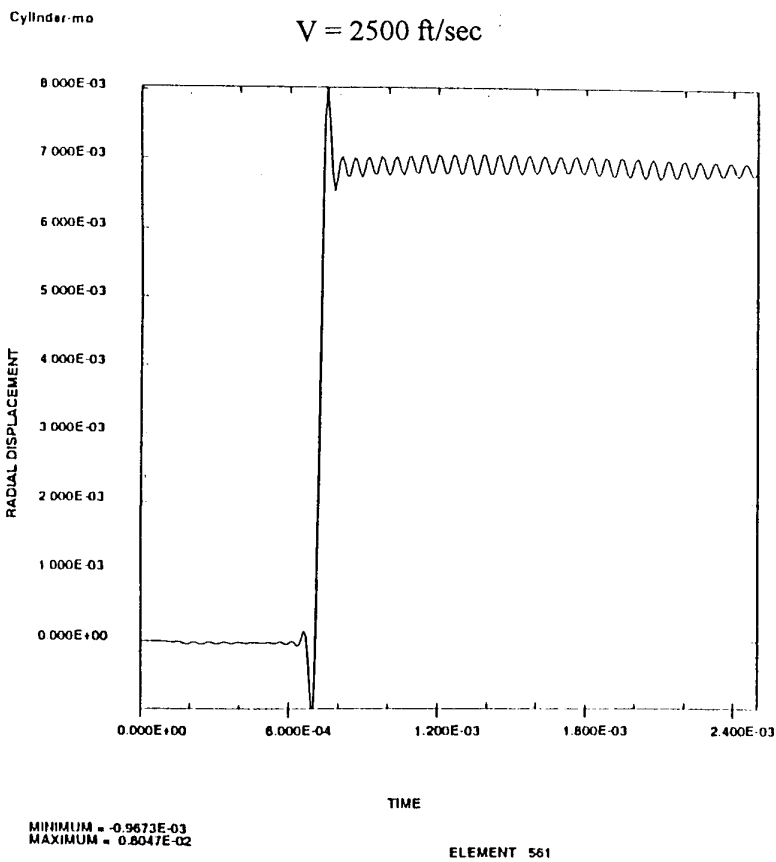

Case 2

$\mathrm{V}=3500 \mathrm{ft} / \mathrm{sec}$

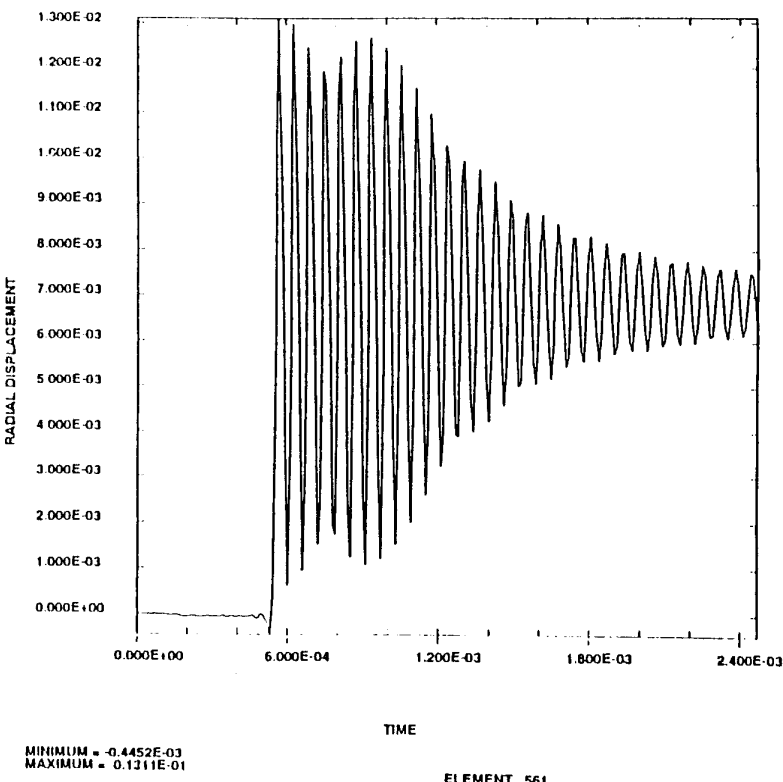

Fig. 5. Radial displacement at the inner radius of composite wrap.

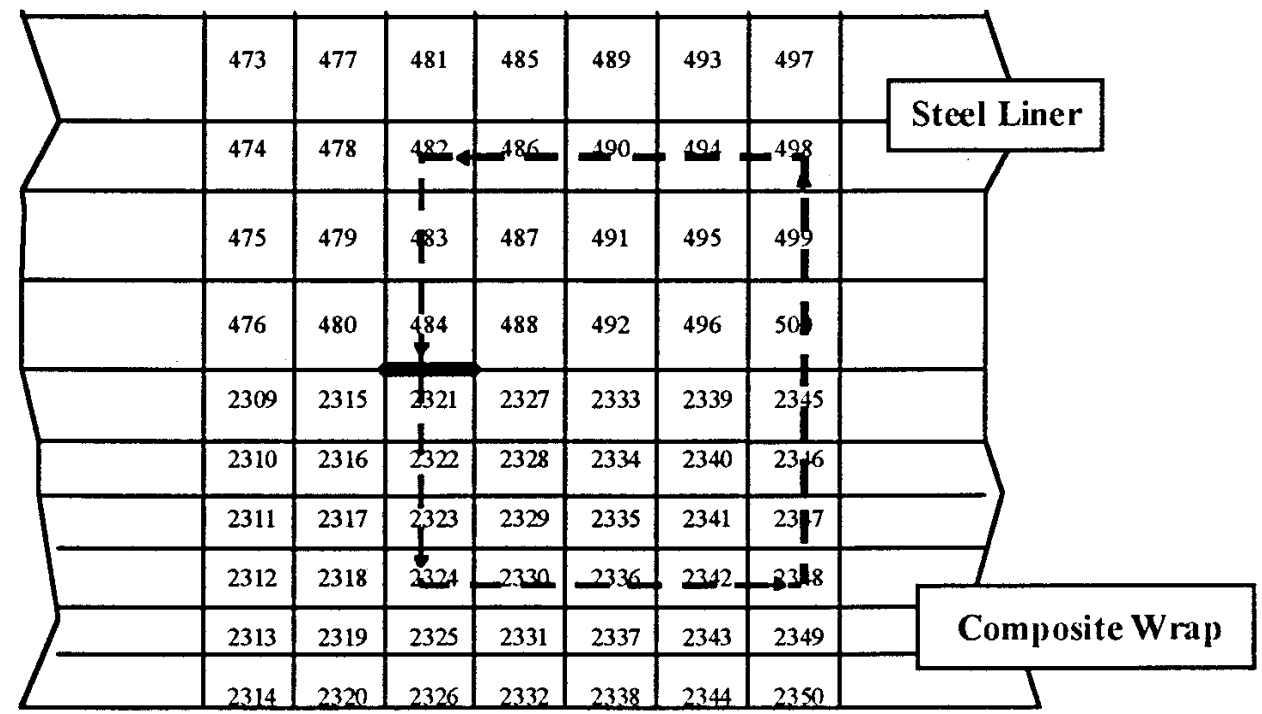

Fig. 6. FEM model and integration path near the crack.

at the center of each element and assumed to be uniform in each element. As the density of the finite element model increases, the integration will approach to an exact solution.

The integration of strain energy density is conservative or path independent. If there is no crack existing, the integration along any closed loop will be equal to zero theoretically. This can be verified numerically by integrating strain energy density along a closed loop using the results from the previous finite element analysis. For a case with cracks, the stress and strain fields surrounding the crack are no longer the same as those 


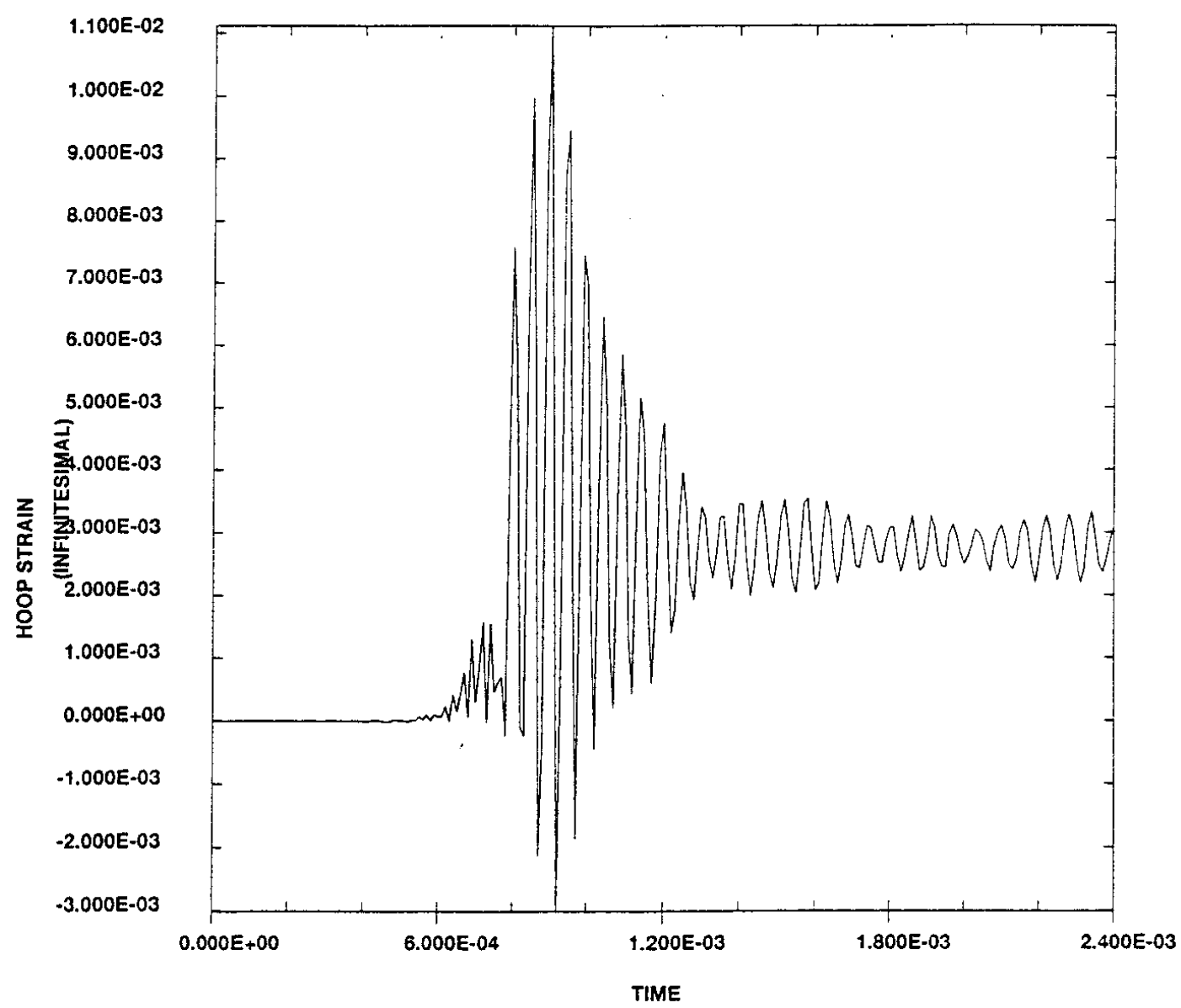

Fig. 7. Hoop strain in an element near the crack.

calculated from a model without cracks. Accordingly, the integration of strain energy density along the path yields to the energy release rate at this specific crack. Furthermore, the integrated energy release rate is determined by the size of crack if the velocity and magnitude of pressure are constant.

Analysis was performed for a model with a crack size of 0.75 in along the interface of composite and liner, which is large enough to cause failure of the cylinder by a rapid crack propagation. The internal pressure is about 6000 psi and the velocity of pressure front is $3500 \mathrm{ft} / \mathrm{sec}$. Figure 8 shows the hoop stress in the steel liner near the crack. It is significantly larger than in a tube without crack. The strain energy density can be calculated from the calculation for specific instant along the selected path. The energy release rate is calculated to be 10 psi-in or $0.01 \mathrm{ksi}$-in from radial, axial, and through thickness shear components. The elastic modulus of the epoxy interface is about $500 \mathrm{ksi}$; accordingly, we can get the combined stress intensity factor as follows

$$
K=\sqrt{K_{I}^{2}+K_{I I}^{2}}=5.0 \mathrm{ksi}-\mathrm{in}^{1 / 2}
$$

The mode III toughness is not included since there is no contribution of in-plane shear components. It is noted that the computed combined stress intensity $(K)$ is much larger than the fracture toughness measured under a static condition. The $K_{1 c}$ is about $1.2 \mathrm{ksi}^{-\mathrm{in}^{1 / 2}}$ and $K_{2 c}$ is about $1.5 \mathrm{ksi}$-in ${ }^{1 / 2}$ for toughened epoxy. The dynamic fracture toughness is not available for the rate of interests generally. It is expected that the fracture toughness will be lower as loading rate increases. Currently, experiments are conducted to measure dynamic toughness for selected materials.

\section{Conclusions}

The dynamic analysis of a composite overwrap tube indicates high magnitude strains and stresses occurring 


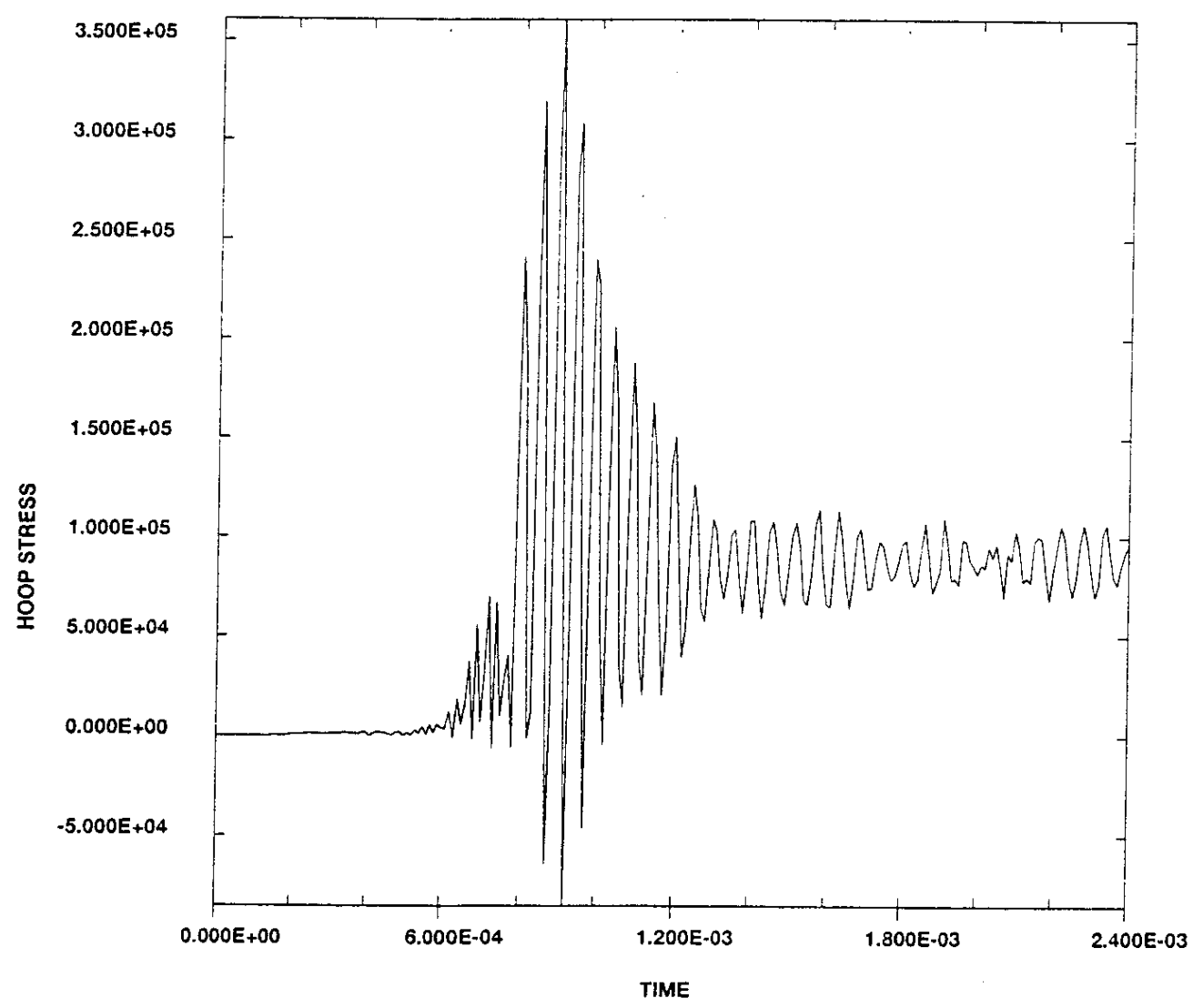

Fig. 8. Hoop stress in an element near the crack.

in the cylinder at the pressure front that traverses along the length of the cylinder. The amplification is caused by the resonance of stress waves attributed to the movement of loading. This dynamic strain effect will potentially cause crack growth and leads to a short life cycle of gun tubes. The propagation of fracture due to the stress waves is particularly critical for lightweight composite cylinders. This is attributed to the low shear and tensile strength at the interface of different materials, thermal degradation, and potential loading rate dependence in polymer composite materials. The proposed analysis provides a straightforward method to evaluate the dynamic response and fracture mechanism in a composite gun barrel, which also exposes the shortcomings of static analysis used in traditional gun tube design.

\section{References}

[1] J.R. Rice, A Path Independent Integral and the Approximate Analysis of Strain Con-centration by Notches and Cracks, Journal of Applied Mechanics (June 1968), 379-386.
[2] G.I. Taylor, Strains in a Gun Barrel Near the Driving Barrel of a Moving Projectile, A.C. 1851/Gn. 104, UK Ministry of Supply, London, England, March 1942.

[3] J.P. Jones and P.G. Bhuta, Response of Cylindrical Shell to Moving Loads, Journal of Applied Mechanics 31, Trans, ASME 86, Series E, March 1964, pp. 105-111.

[4] S. Tang, Dynamic Response of a Tube Under Moving Pressure, Journal of the Engineering Mechanics Division Proceedings of the ASCE (October 1965), 97-122.

[5] H. Reismann, Response of a Prestressed Cylindrical Shell to Moving Pressure Load, Development in Mechanics, Solid Mechanics - Proceedings of the Eighth Midwestern Mechanics Conference, Pergamon Press, Part II, Vol. 2, 1965, pp. 349363.

[6] T.E. Simkins, Response of Flexural Waves in Gun Tubes, Tech. Report ARCCB-TR-87008, US Army ARDEC, Benet Weapons Laboratory, Watervliet, NY, July 1987.

[7] D.A. Hopkins, Predicting Dynamic Strain Amplification by Coupling a Finite Element Structural Analysis Code with a Gun Interior Ballistic Code, BRL-TR-3269, US Army Ballistic Research Laboratory, APG, MD, September 1991.

[8] J.T. Tzeng and D.A. Hopkins, Dynamic Response of Composite Cylinders Subjected to a Moving Internal Pressure, Journal of Reinforced Plastics and Composites 15(11) (November 1996), 1088-1105.

[9] J.O. Hallquist, User Manual for DYNA2D - An Explicit TwoDimensional Hydrodynamic Finite Element Code With Inter- 
active Rezoning and Graphical Display, Lawrence Livermore National Laboratory, 1987.

[10] S.N. Atluri, Computational Methods in Mechanics of Fracture, (Chapter 5), Springer-Verlag, NY, 1985,

[11] J.F. Doyle and T.N. Farris, Interaction of Longitudinal Waves With a Lengthwise Crack in a Beam, ESM Proceedings,
Portland, June 1988, pp. 1253-1260.

[12] A. Alexander and J.T. Tzeng, Three Dimensional Effective Properties of Composite Materials for Finite Element Applications, Journal of Composite Materials 31(5) (1997), 466485 . 

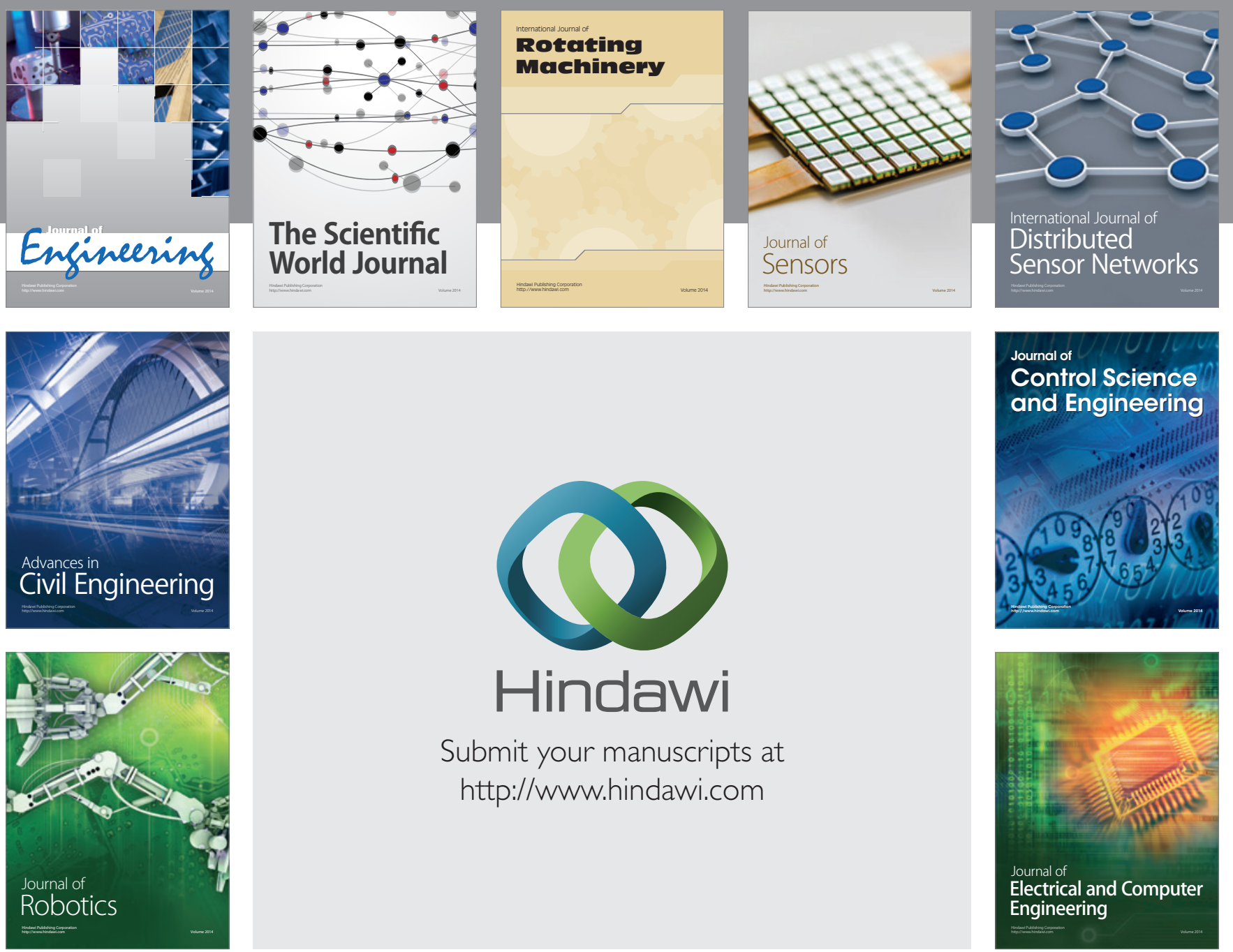

Submit your manuscripts at

http://www.hindawi.com
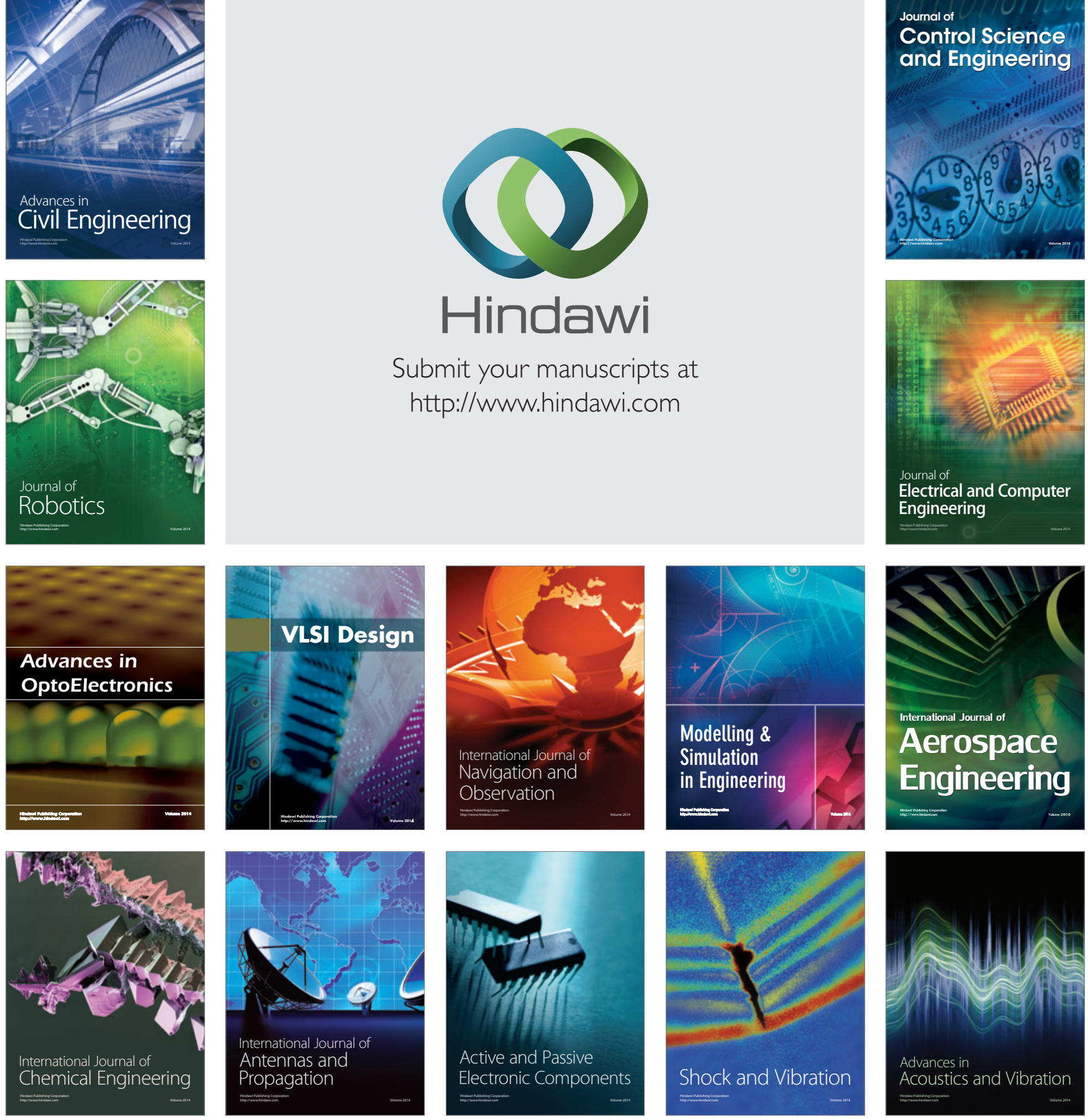